\title{
A Novel Design for a High Power Superconducting Delay Line
}

Yu Ju Chen, George J. Caporaso

This paper was prepared for submittal to the

1997 Particle Accelerator Conference

Vancouver, BC, Canada

May 12-16, 1997

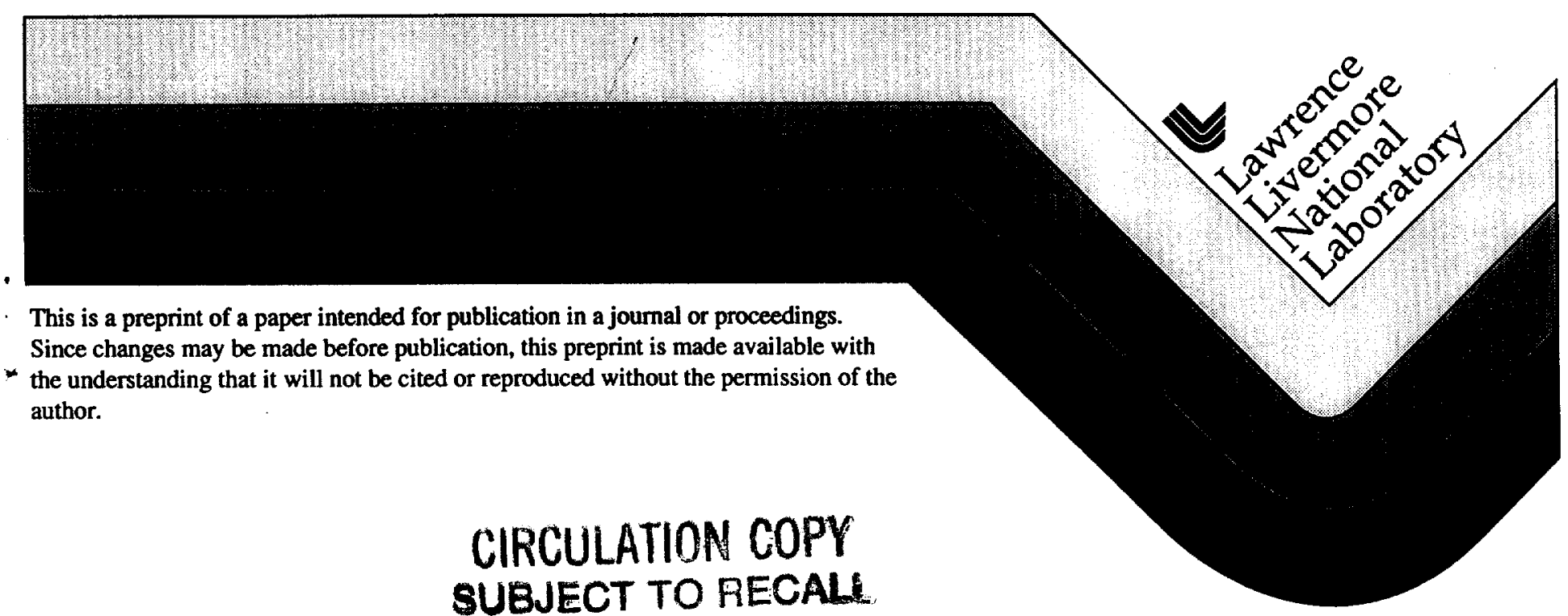

IN TWO WEEKS 


\section{DISCLAIMER}

This document was prepared as an account of work sponsored by an agency of the United States Government. Neither the United States Government nor the University of California nor any of their employees, makes any warranty, express or implied, or assumes any legal liability or responsibility for the accuracy, completeness, or usefulness of any information, apparatus, product, or process

disclosed, or represents that its use would not infringe privately owned rights. Reference herein to any specific commercial product, process, or service by trade name, trademark, manufacturer, or otherwise, does not necessarily constitute or imply its endorsement, recommendation, or favoring by the United States Government or the University of California. The views and opinions of authors expressed herein do not necessarily state or reflect those of the United States Government or the University of California, and shall not be used for advertising or product endorsement purposes. 


\title{
A NOVEL DESIGN FOR A HIGH POWER SUPERCONDUCTING DELAY LINE
}

\author{
Yu Ju (Judy) Chen and George J. Caporaso \\ Lawrence Livermore National Laboratory
}

\begin{abstract}
Potential designs for a high power superconducting delay line of approximately $10 \mathrm{~ms}$ duration are described. The transmitted signal should have low dispersion and little attenuation to recapture the original signal. Such demands cannot be met using conventional metal conductors. This paper outlines a proposal for a new transmission line design using low temperature superconducting material which meets system specifications. The $25 \mathrm{~W}$ line is designed to carry pulsed signals with an approximate rise time of 8nsec and a maximum voltage magnitude of $25 \mathrm{kV}$. Predicted electrical design and performance of the line will be presented.
\end{abstract}

\section{APPLICATION OF THE LINE}

The line will be used to provide transit time isolation of the reflections expected to be generated in the kicker of an AHF accelerator [1]. The kicker will operate with beam currents of 3 to $6 \mathrm{kA}$ and will launch beam-induced voltages up the transmissions lines connecting it to its pulsers. The most straightforward way to eliminate the effects of these reflections is to transit time isolate the kicker from the pulser. Thus the $10 \mu \mathrm{sec}$ line will actually allow a $20 \mu \mathrm{sec}$ operational interval.

\section{WHY USE SUPERCONDUCTORS?}

\subsection{Attenuation}

The general expression for attenuation is

$$
\alpha=\frac{1}{2} \frac{R_{s}}{Z_{0}} \sqrt{\frac{\varpi}{2}}
$$

where Rs is the skin-effect impedance dependent on the geometry and resistivity of the structure [2].

For a coax where $a$ and $b$ are the inner and outer diameter, $R_{s}$ is derived to be

$$
R_{s}=\frac{\sqrt{j \sigma} \mu \Gamma_{r}^{2}}{2 \pi}\left[\begin{array}{l}
\frac{1}{a^{2}}-\frac{1}{b^{2}}+\frac{1}{2 \Gamma, a} \frac{I_{0}\left(a / \Gamma_{r}\right)+I_{2}\left(a / \Gamma_{r}\right)}{I_{1}\left(a / \Gamma_{r}\right)}+ \\
\frac{1}{2 \Gamma_{r} b} \frac{K_{0}\left(b / \Gamma_{r}\right)+K_{2}\left(b / \Gamma_{r}\right)}{K_{1}\left(b / \Gamma_{r}\right)}
\end{array}\right]
$$

where $I_{n}$ and $K_{n}$ are the modified Bessel functions of the first and second kind. $\Gamma_{r}$ is associated with the skin depth and is

$$
\Gamma_{r}=\left\{\begin{array}{l}
1 / \sqrt{j \omega \mu \sigma} \\
1 / \sqrt{j \omega \mu \sigma+1 / \lambda^{2}}
\end{array}\right.
$$

normal conducting metal

$$
\text { Fpe I superconductor }
$$

where $\lambda$ is the penetration depth of the superconductor. At high frequencies the expression simplifies to a more familiar expression [2][3].

$$
R_{s}=\frac{\sqrt{j \varpi} \mu}{2 \pi} \Gamma_{r}\left(\frac{1}{a}+\frac{1}{b}\right) .
$$

Suppose the line was made of copper $(\sigma=$ $\left.5.92 \mathrm{e} 7(\Omega-\mathrm{m})^{-1}\right)$ and the inner and outer diameters were $2 \mathrm{~cm}$ and $3 \mathrm{~cm}$ respectively. With an $8 \mathrm{nsec}$. rise time, we used $125 \mathrm{MHz}$ to be the highest critical frequency, which is about 3 times that of the roll-off frequency. For $125 \mathrm{MHz}, \alpha=7.658 \mathrm{e}-4 / \mathrm{m}$. For a $3 \mathrm{~km}$ line, this corresponds to a power attenuation of $\left(1-\mathrm{e}^{-2 \alpha \cdot 3000 \mathrm{~m}}\right)=98.9 \%$. The signal has virtually disappeared!

On the other hand, for the same set of dimensions, a niobium $\left(\lambda=39 \mathrm{nml}, \sigma=8.03 \mathrm{e} 6(\Omega-\mathrm{m})^{-1}\right)$ line yields an $\alpha$ of approximately $5.1025 \mathrm{e}-6(1+j) / \mathrm{m}$. The imaginary term contributes to the inductance of the line. The power attenuation is now $3 \%$ at $125 \mathrm{MHz}$ which is an upper limit. This is the most compelling reason to use a superconducting line.

\section{DESIGN OF A SUPERCONDUCTING LINE}

The size of the transmission line is fundamentally limited by the critical field of the superconducting material. Of the type I conductors, niobium ( $\mathrm{Nb}$ ) is the most generous material since it has the highest critical $B$-field of $.206 \mathrm{~T}$ [2] at $T=0 \mathrm{~K}$.

Assuming the distribution for the critical field to be $B_{c}(T)=B_{c}(0)\left[1-\left(T / T_{c}\right)^{2}\right]$, the critical field at $4.2 \mathrm{~K}$ (the usual operating point of liquid helium) is .1635T. The $B$ field of a structure can be written as $B=g I$ where $g$ is a geometry factor. The condition on $g$ is therefore $g \leq B_{c} / I$

\subsection{Coaxial line}

For a concentric coax, $g=\mu / 2 \pi r$ where $r$ is either the inner or outer radius of the line. This yields the condition that $r>\mu I / 2 \pi B_{c}$. If one wants to operate at $20 \%$ of maximum capacity, then $a=.2 \mu L / 2 \pi B_{c}$ where $a$ is the inner radius. The current on the line can be as high as 1kA. This requires that $a=6.12 \mathrm{~mm}$.

1 This work was performed under the auspices of the U.S. Department of Energy by the Lawrence Livermore National Laboratory under Contract No. W-7405-Eng-48. 
Since the layer of niobium only needs to be much greater than the penetration depth $\lambda$, a thin coat of approximately .5um will be deposited on a copper substrate as shown in Fig. 1. The need for a metal substrate will be discussed in section 4 . The area between the two conductors will be filled with a material that may or may not have dielectric properties. The inner conductor should be hollow to allow helium to flow through the tube.

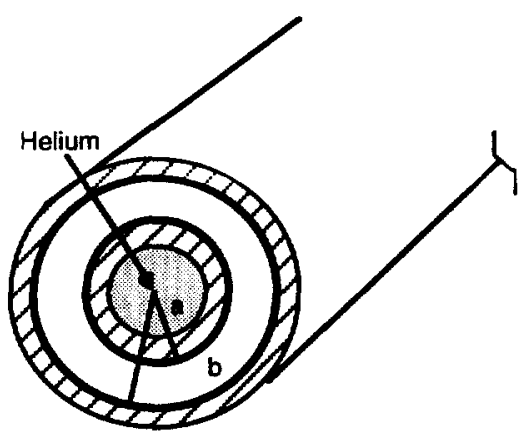

Fig. 1. Coaxial line with $\mathrm{Nb}$ layers deposited on copper tubes

Design 1: The outer conductor radius, for an impedance of $25 \Omega$, is $9.28 \mathrm{~mm}$. Design 2: If one puts in a dielectric material of $\varepsilon_{r}=10$, the outer radius goes up to $22.85 \mathrm{~mm}$. The advantage of the dielectric is that the length of the line is reduced by a factor of $1 / \sqrt{ } 10$ which yields a length of $948 \mathrm{~m}$.

\subsection{Stripline}

For manufacturing considerations, it may be easier to fabricate striplines instead of coaxial lines. The solution for the stripline problem, both open and closed, has been derived by Primozich [4]. As it turns out, a stripline with a dielectric filler is too dispersive (see section 5). Therefore, the design cannot have a dielectric material. Design 3: The stripline dimensions are shown in Fig. 2.

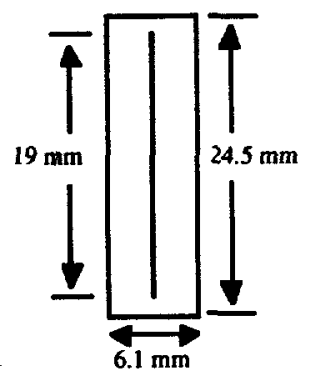

Fig. 2. Dimensions of stripline design

\subsection{Sheath helix}

Sheath helix lines are like coaxial lines, only the inner conductor is wrapped at an angle. These are slow wave structures and ideal for a delay line. However, they are more dispersive than regular coax. Fig. 3 shows two sets of two overlayed $10 \mathrm{kV}$ pulses. The solid line is the actual signal at the output of the line and the dotted is the output had there been no dispersion. These plots are derivations based on Lund [5] and are for uncurved lines only.

Design 4: Fig. 3(a) corresponds to a line with $\varepsilon_{r}=1$, $b / a=1.15$, $p$ (pitch) $/ a=2.1$, and $v / c=.336$. The line length is reduced to about $1 / 3$ of the original length. Part (b) corresponds to $\varepsilon_{r}=3, b / a=1.4, p / a=2.75$, and $v / c=.264$. The second line yields a slightly slower wave but at the cost of increased dispersion as evident by the wiggles at the rising part of the pulse.
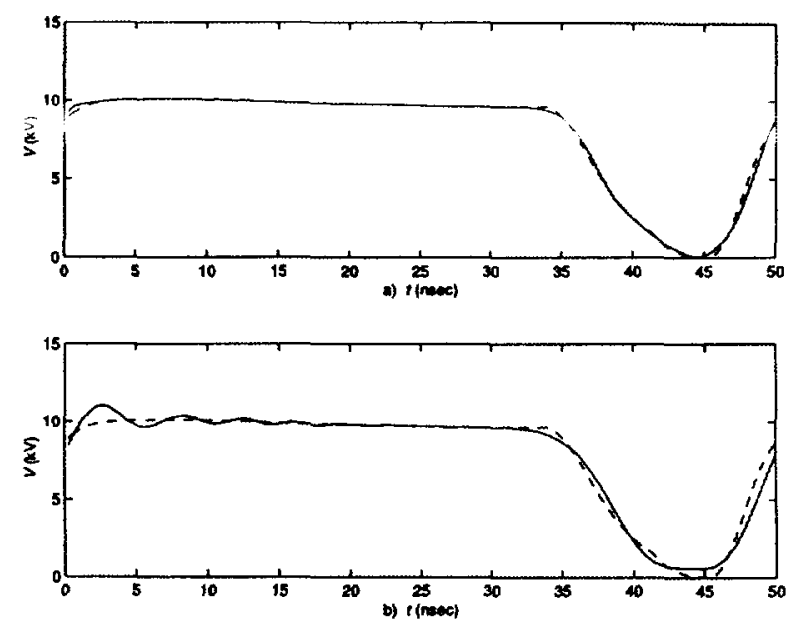

Fig. 3. a) $-10 \mathrm{kV}$ pulse at output of line for sheath helix without dielectric, - - - same pulse if line were non-dispersive

b) same as a) except line has dielectric. Wiggles indicate more dispersion

The sheath helix line is presented here as a backup design in case the stripline is not easy to manufacture because of it's length or the dielectric used for the coaxial line proves to be lossy or difficult to work with.

\section{THERMAL STABILITY}

Niobium can be deposited on copper as illustrated in Fig. 1 where the fields and currents flow. The copper acts as a stabilizer in case the superconducting portion exceeds critical current and quenches.

The condition for thermal stability ${ }^{2}$ is

$$
\alpha=\frac{\rho \lambda^{2} J_{c}^{2}}{(1-\lambda)} \frac{A}{\operatorname{Ph}\left(\theta_{c}-\theta_{0}\right)}<1
$$

where $\rho$ (resistivity of copper) $=1 \mathrm{e}-10 \Omega \mathrm{m}, \lambda=$ fraction of superconductor, $J_{c} \equiv$ superconducting critical current density, $P \equiv$ cooled perimeter, $h$ (heat transfer coefficient) $=1 \mathrm{e} 3 \mathrm{~W} / \mathrm{m}^{2}, A \equiv$ cross-sectional area of conductor, $\theta_{\mathrm{c}}$ (critical temperature $)=9.25 \mathrm{~K}$, and $\theta_{0}$ (bath temperature $)=4.2 \mathrm{~K}$.

For the coaxial designs, the inner conductor should have a copper thickness of at least $.37 \mathrm{~mm}$. For the outer conductor, the thickness should be at least $.32 \mathrm{~mm}$ for both designs 1 and 2 .

${ }^{2}$ For dc conditions. See Wilson [6] p. 92. 
The stripline (design 3) has an inner strip that is surrounded by filler material and has no contact with helium. The shield, on the other hand, is in contact with helium. The calculated thickness of the copper is $.33 \mathrm{~mm}$.

\section{DISPERSION}

The previous section has already shown that dispersion from skin depth is negligible for a superconducting line. The dispersion on the superconducting transmission line, instead, is caused by wrapping the long line onto either a drum or into a pancake shape. The electromagnetic problem for a curved coax has been solved in [7].

The propagation constant for a curved coax is as follows

$$
\gamma^{2}=\gamma_{0}^{2}\left[1+\frac{A_{2}}{R^{2}}+O\left(\frac{1}{R^{4}}\right)\right]
$$

where $\gamma_{0}=\omega \sqrt{ } \mu \varepsilon, A_{2}=A \omega^{2}+B$, and $R=R(s)$. The constants $A$ and $B$ can be found in [7]. For a pancake design, the outer cable radius is, as a function of $s$, the total length, and $R_{0}$, the inner radius,

$$
R(s)=\sqrt{R_{0}{ }^{2}+\frac{2 b s}{\pi}}
$$

If one wants to find the total dispersion at the end of the line, the propagation constant would need to be integrated over the length of the line since it varies with $s$. In other words,

$$
e^{-j x} \rightarrow e^{-j \int d s^{\prime}}
$$

Therefore, the phase shift of the signal at the end of the line is

$$
\Delta \phi=-\frac{A_{2}(\varpi)}{2} \gamma_{0} \int_{0}^{s} \frac{d s^{\prime}}{R^{2}\left(s^{\prime}\right)}=-\gamma_{0} \frac{\pi A_{2}}{4 b} \ln \left(1+\frac{2 b s}{\pi R_{0}^{2}}\right)
$$

The phase velocity and $\Delta \phi$ for design 2 is plotted in Fig. 4. As one can see in Fig. 4b, the phase distortion
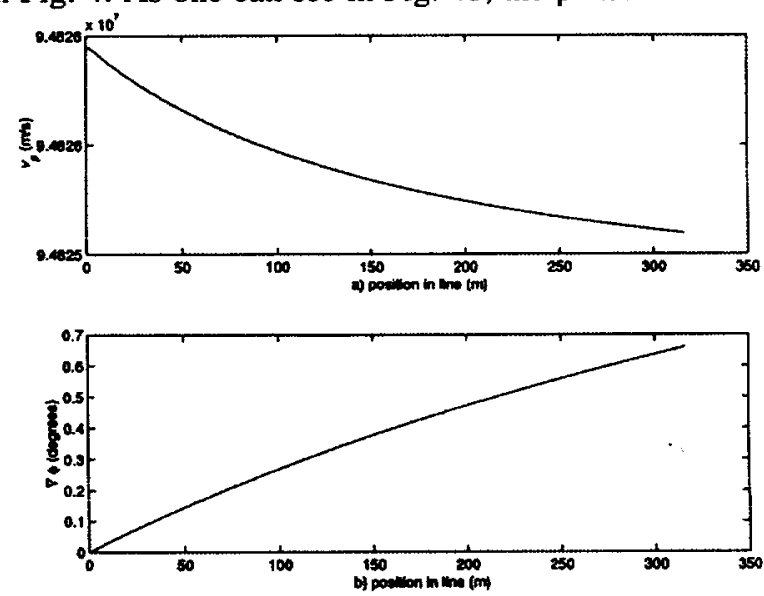

Fig. 4. a) phase velocity as function of position in a pancake-wound coax line

b) it's corresponding phase shift at $125 \mathrm{MHz}$

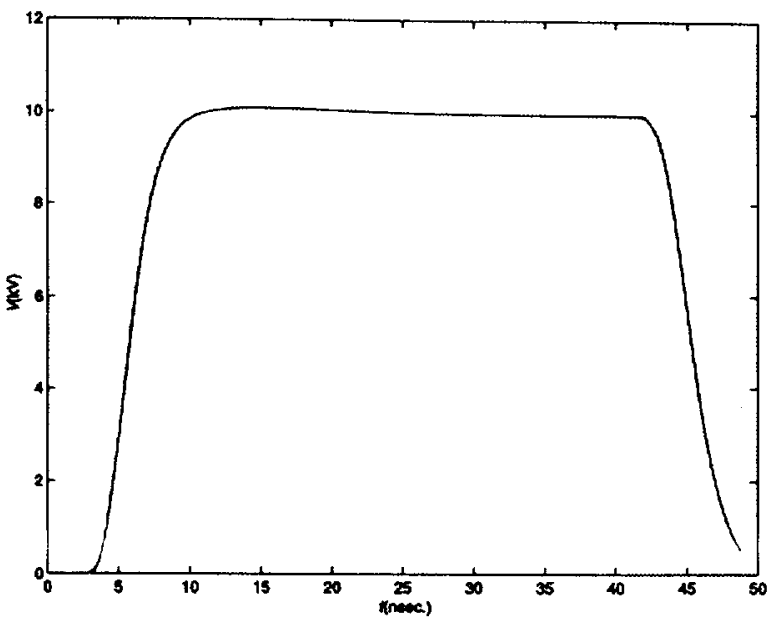

Fig. 5. Design 2 placed in kicker dynamics program produced almost exact replication of original pulse (figure actually has 2 overlapping curves)

is less than $1^{\circ}$ at the end of the line. The striplines were treated as equivalent coax lines to estimate the dispersion due to bending.

The parameters for designs 1-3 were run through a kicker dynamic code to examine the acceptability of the output signal. All 3 designs produced clean output signals. An output plot of design 2 is shown in Fig. 5 where $R_{0}=1 \mathrm{~m}$.

\section{CONCLUSION}

Attenuation in long transmission lines made use of superconductors a necessity. The attenuation in the superconducting line is very small, with joints connecting sections of the line contributing to most of it. Dispersion, on the other hand, can arise simply from the geometry of the line. Curved lines and helix lines are subject to dispersion. Two coaxial lines, one stripline, and a sheath helix design were presented. The challenge of fabricating the line will determine which design is ultimately adopted.

\section{REFERENCES}

[1] G. J. Caporaso, "Linear Induction Accelerator Approach for Advanced Radiography," these proceedings.

[2] R. E. Matick, "Transmission Lines for Digital and Communication Networks," reissued by IEEE PRESS, 1995

[3] T. Van Duzer, C. W. Turner, "Principles of Superconductive Devices and Circuits," published by Edward Arnold, Ltd., 1981.

[4] F. G. Primozich, AIEE, May 1955.

[5] C. O. Lund, RCA Review, March 1950.

[6] M. N. Wilson, "Superconducting Magnets," published by Oxford University Press, 1983.

[7] J. J. Krempasky, IEEE Transactions-MTT, v38, n6, June 1990. 


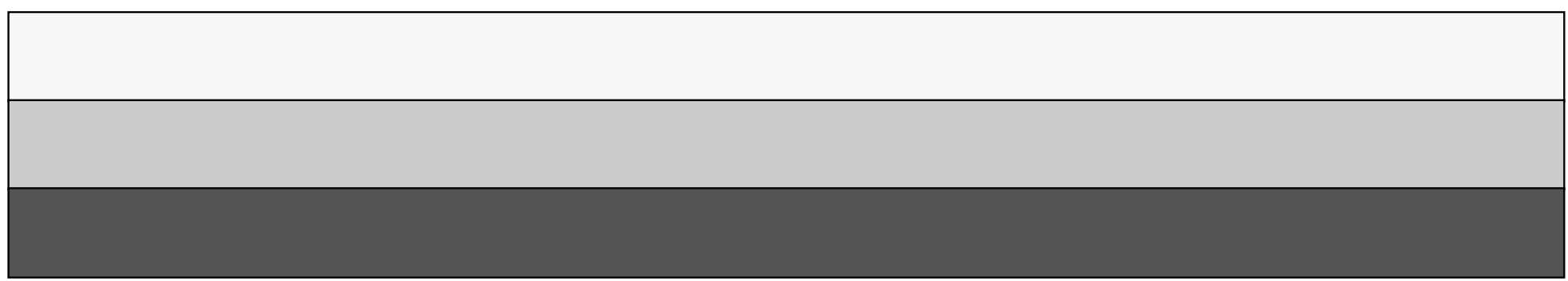

